\title{
Human adipose tissue-derived multilineage progenitor cells exposed to oxidative stress induce neurite outgrowth in PC12 cells through p38 MAPK signaling
}

Mariko Moriyama ${ }^{1,2+}$, Hiroyuki Moriyama ${ }^{1{ }^{*}+}$, Ayaka Ueda ${ }^{1}$, Yusuke Nishibata ${ }^{1}$, Hanayuki Okura² ${ }^{2}$ Akihiro Ichinose ${ }^{3}$, Akifumi Matsuyama ${ }^{2}$ and Takao Hayakawa ${ }^{1}$

\begin{abstract}
Background: Adipose tissues contain populations of pluripotent mesenchymal stem cells that also secrete various cytokines and growth factors to support repair of damaged tissues. In this study, we examined the role of oxidative stress on human adipose-derived multilineage progenitor cells (hADMPCs) in neurite outgrowth in cells of the rat pheochromocytoma cell line (PC12).

Results: We found that glutathione depletion in hADMPCs, caused by treatment with buthionine sulfoximine (BSO), resulted in the promotion of neurite outgrowth in $\mathrm{PC} 12$ cells through upregulation of bone morphogenetic protein 2 (BMP2) and fibroblast growth factor 2 (FGF2) transcription in, and secretion from, hADMPCs. Addition of Nacetylcysteine, a precursor of the intracellular antioxidant glutathione, suppressed the BSO-mediated upregulation of BMP2 and FGF2. Moreover, BSO treatment caused phosphorylation of p38 MAPK in hADMPCs. Inhibition of p38 MAPK was sufficient to suppress BMP2 and FGF2 expression, while this expression was significantly upregulated by overexpression of a constitutively active form of MKK6, which is an upstream molecule from p38 MAPK.
\end{abstract}

Conclusions: Our results clearly suggest that glutathione depletion, followed by accumulation of reactive oxygen species, stimulates the activation of p38 MAPK and subsequent expression of BMP2 and FGF2 in hADMPCs. Thus, transplantation of hADMPCs into neurodegenerative lesions such as stroke and Parkinson's disease, in which the transplanted hADMPCs are exposed to oxidative stress, can be the basis for simple and safe therapies.

Keywords: Human adipose-derived multilineage progenitor cells, Adult stem cells, Reactive oxygen species, p38 MAPK, Neurite outgrowth, BMP2, FGF2, Neurodegenerative disorders

\section{Background}

Mesenchymal stem cells (MSCs) are pluripotent stem cells that can differentiate into various types of cells [1-6]. These cells have been isolated from bone marrow [1], umbilical cord blood [2], and adipose tissue [3-6] and can be easily obtained and expanded ex vivo under appropriate culture conditions. Thus, MSCs are an attractive material for cell therapy and tissue engineering.

\footnotetext{
* Correspondence: moriyama@phar.kindai.ac.jp

${ }^{\dagger}$ Equal contributors

${ }^{1}$ Pharmaceutical Research and Technology Institute, Kinki University, 3-4-1 Kowakae, Higashi-Osaka, Osaka 577-8502, Japan

Full list of author information is available at the end of the article
}

Human adipose tissue-derived mesenchymal stem cells, also referred to as human adipose tissue-derived multilineage progenitor cells (hADMPCs), are especially advantageous because they can be easily and safely obtained from lipoaspirates, and the ethical issues surrounding other sources of stem cells can be avoided [4-6]. Moreover, hADMPCs have more pluripotent properties for regenerative medical applications than other stem cells, since these cells have been reported to have the ability to migrate to the injured area and differentiate into hepatocytes [4], cardiomyoblasts [5], pancreatic cells [7], and neuronal cells [8-10]. In addition, it is known that hADMPCs secrete a wide variety of cytokines and




growth factors necessary for tissue regeneration including nerve growth factor (NGF), brain-derived neurotrophic factor (BDNF), fibroblast growth factors (FGFs), vascular endothelial growth factor (VEGF) and hepatocyte growth factor (HGF) [11-14].

Recently, several groups have reported that hADMPCs facilitate neurological recovery in experimental models of stroke [9,10,15] and Parkinson's disease [16]. Despite the superiority of hADMPCs over other stem cells, the potential use of hADMPCs for the treatment of these neurodegenerative disorders has not been fully investigated. It has been reported that administration of
hADMPCs in animal models of acute ischemic stroke markedly decreased brain infarct size, improved neurological function by enhancing angiogenesis and neurogenesis, and showed anti-inflammatory and antiapoptotic effects $[9,10]$. These effects were due in part to increased secretion levels of VEGF, HGF and bFGF under hypoxic conditions [13], indicating the role of hADMPCs in reducing the severity of hypoxia-ischemic lesions.

In addition to hypoxic stress, ischemic lesions are generally subject to inflammation, which leads to the generation of reactive oxygen species (ROS) $[17,18]$. ROS are
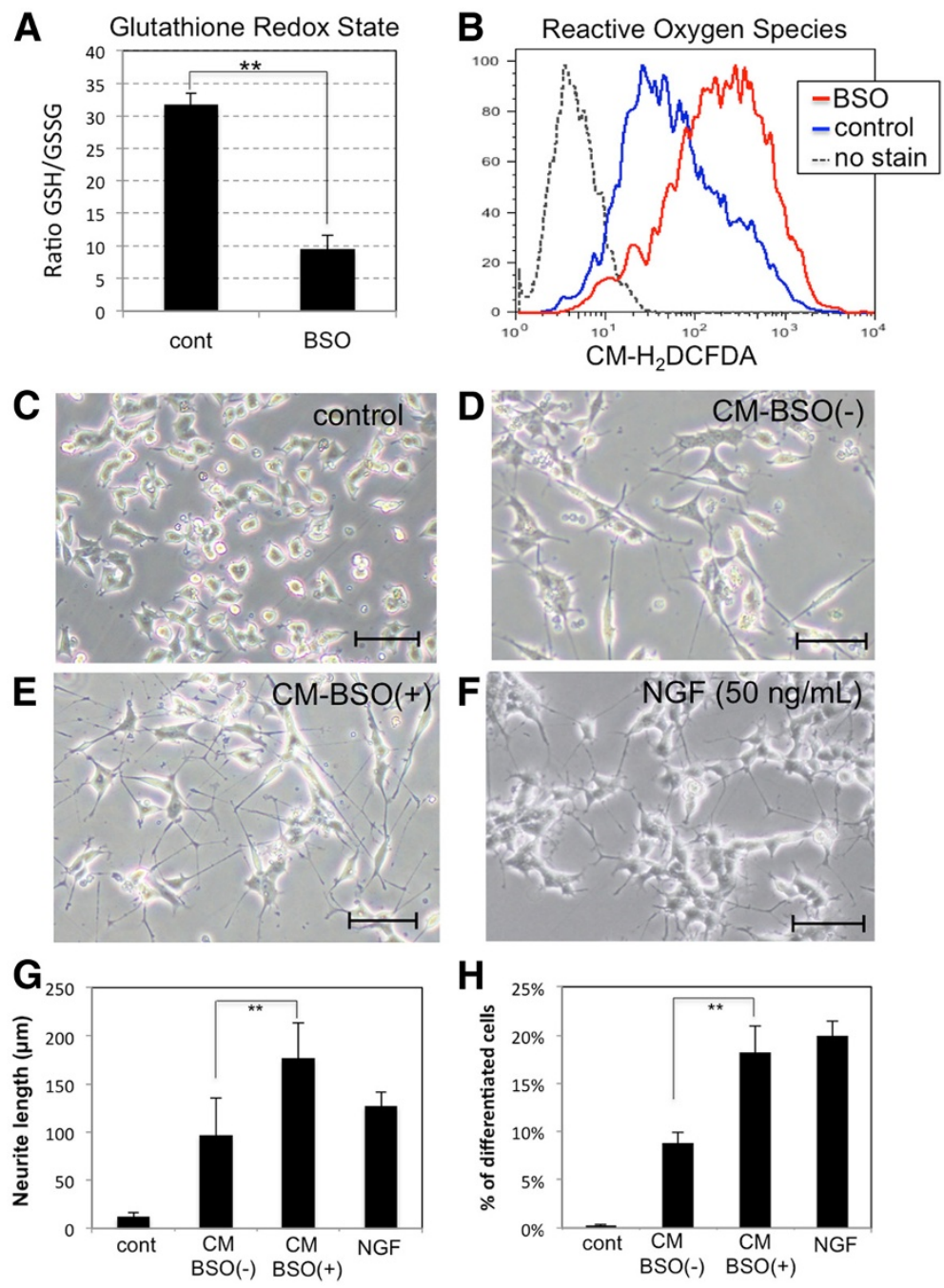

Figure 1 Conditioned medium from hADMPCs exposed to oxidative stress induces neurite outgrowth in PC12 cells. (A, B) Decrease of the reduced/oxidized glutathione ratios and increase in the intracellular ROS levels in hADMPCs treated with BSO. hADMPCs were treated with $1 \mathrm{mM}$ BSO for $16 \mathrm{~h}$, and cellular GSH/GSSG levels $(\mathbf{A})$ or ROS $\left(\mathrm{H}_{2} \mathrm{O}_{2}\right)$ levels (B) were analyzed. (C-G) Induction of neurite outgrowth in PC12 cells by conditioned medium from BSO-treated hADMPCs. PC12 cells were induced to differentiation by changing medium to differentiation medium alone (C), CM-BSO (-) (D), CM-BSO (+) (E), or differentiation medium with NGF (50 ng/mL) (F) for 2 days. Scale bars, $200 \mu$ m. (G) One hundred individual neurites were measured in each sample using Dynamic Cell Count Analyzer BZ-H1C (Keyence, Osaka, Japan) and average neurite length was calculated. **, P $<0.01$ (Student's $t$ test). (H) Percentage of neurite-bearing PC12 cells. A cell was scored positive for bearing neurites if it has a thin neurite extension that is double the length of the cell body diameter. A total of 500-600 cells in each sample were counted. ${ }^{* *}$, $P<0.01$ (Student's t test). 
generated as a natural byproduct of normal aerobic metabolism, and mitochondrial respiration, together with oxidative enzymes such as plasma membrane oxidase, is considered to be the major intracellular source of ROS production [19]. Although appropriate levels of ROS play an important role in several physiological processes, oxidative damage initiated by excessive ROS causes many pathological conditions including inflammation, atherosclerosis, aging, and cancer. Neuronal cells are especially vulnerable to oxidative stress, and numerous studies have examined the crucial roles of oxidative stress in neurodegenerative disorders such as stroke $[17,18]$, Alzheimer's disease [20,21], and Parkinson's disease $[22,23]$. In these diseases, microglia, the macrophages of the central nervous system (CNS), are activated in response to a local inflammation [24] and generate large amounts of reactive oxygen and nitrogen species, thereby exposing nearby neurons to stress $[18,25]$. Thus, the influence of oxidative stress generated by neurodegenerative lesion on hADMPCs needs to be further studied.

In this study, we examined the role of oxidative stress on hADMPCs in neurite outgrowth in cells of the rat pheochromocytoma cell line (PC12). Upon treatment with buthionine sulfoximine (BSO), an inhibitor of the rate-limiting enzyme in the synthesis of glutathione, hADMPCs accumulated ROS, which resulted in the upregulation of expression levels of the neurotrophic factors BMP2 and FGF2. Our present data thus provide new insights into understanding the mechanism of how hADMPCs exposed to oxidative stress contribute to neurogenesis, and this may explain the effects of stem cell transplantation therapy with hADMPCs in treating ischemic stroke.

\section{Results}

hADMPCs exposed to oxidative stress stimulate neurite outgrowth in PC12 cells

hADMPCs were treated with $1 \mathrm{mM}$ BSO for $24 \mathrm{~h}$; a group of hADMPCs that were not given any treatment was used as the control group. As shown in Figure 1A and B, BSO treatment resulted in significant reduction of intracellular reduced glutathione levels, followed by accumulation of intracellular reactive oxygen species (ROS) in hADMPCs. To investigate whether accumulation of ROS affects secretion of cytokines from hADMPCs, conditioned medium from BSO-treated (CM-BSO (+)) or BSO-untreated (CMBSO (-)) hADMPCs was added to PC12 cells. As expected, addition of NGF significantly induced neurite outgrowth in the PC12 cells (Figure 1F, G, H). hADMPCs, like other mesenchymal stem cells derived from bone marrow or adipose tissue, may secrete many cytokines including NGF, BDNF and FGF2, and this may account for the slight induction of neurite outgrowth seen in the CM-
BSO (-) treated cells (Figure 1D, G, H). In contrast, the number and length of neurite outgrowth of PC12 cells in CM-BSO (+) (Figure 1E) was markedly enhanced compared with those in CM-BSO (-) (Figure 1D, E, G, H).

\section{Conditioned medium from BSO-treated hADMPCs} activates Erk1/2 MAPK and Smad signaling in PC12 cells

To investigate which intracellular signaling pathways were involved in the neurite outgrowth of PC12 cells in CM-BSO (+), we used western blotting to determine the phosphorylation levels of Erk1/2 MAPK, p38 MAPK, Smad1/5/8 and Akt in PC12 cells in various culture conditions. NGF significantly activated Erk1/2 MAPK and Akt signaling pathway (Figure 2). In contrast, Erk $1 / 2$ MAPK was not activated in PC12 cells exposed to CMBSO (-), while an increase in phosphorylated Smad1/5/8 was observed. Interestingly, CM-BSO (+) treatment led to both a significant increase in Smad1/5/8 phosphorylation levels as well as activation of the Erk1/2 MAPK

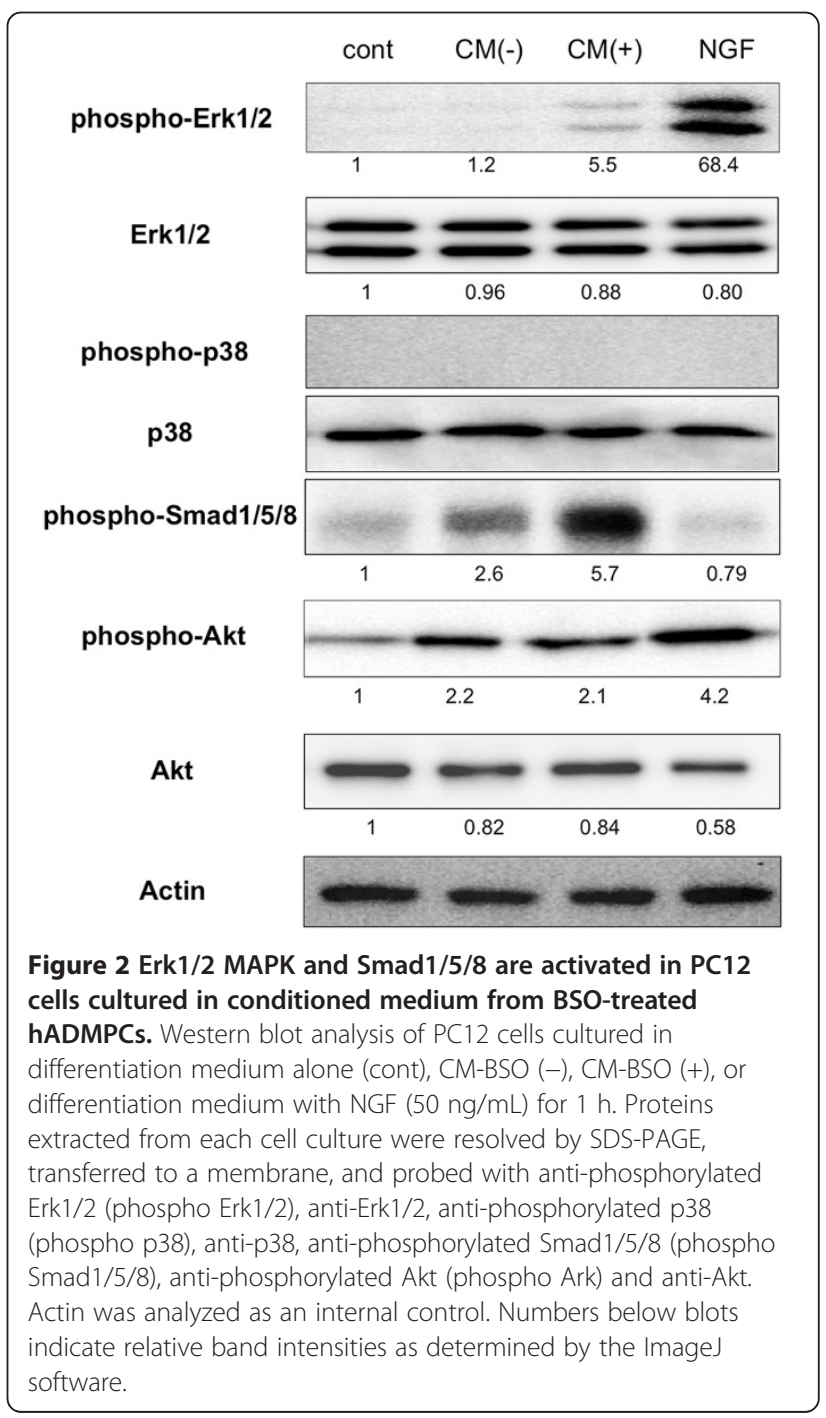


signaling pathway in PC12 cells (Figure 2). Akt was 2fold activated in both CM-BSO (-) and CM-BSO (+) treated PC12 cells, but no significant difference between the 2 groups was observed.

\section{FGF2 and BMP2 are upregulated through p38 MAPK} signaling in hADMPCs exposed to oxidative stress We next examined which growth factors or cytokines from BSO-treated hADMPCs were involved in stimulation of neurite outgrowth. We found that both mRNA (Figure $3 \mathrm{~A}$ and $\mathrm{B}$ ) and protein (Figure $3 \mathrm{C}$ and $\mathrm{D}$ ) levels for BMP2 and FGF2 were markedly increased in hADMPCs treated with BSO. To determine if this upregulation was caused by ROS, all cells were exposed to the antioxidant $N$-acetylcysteine (NAC). As we expected, addition of NAC to BSO-treated hADMPCs reduced the expression levels of BMP2 and FGF2 to control levels (Figure 3E and F). As BMP2 together with FGF2 has


H
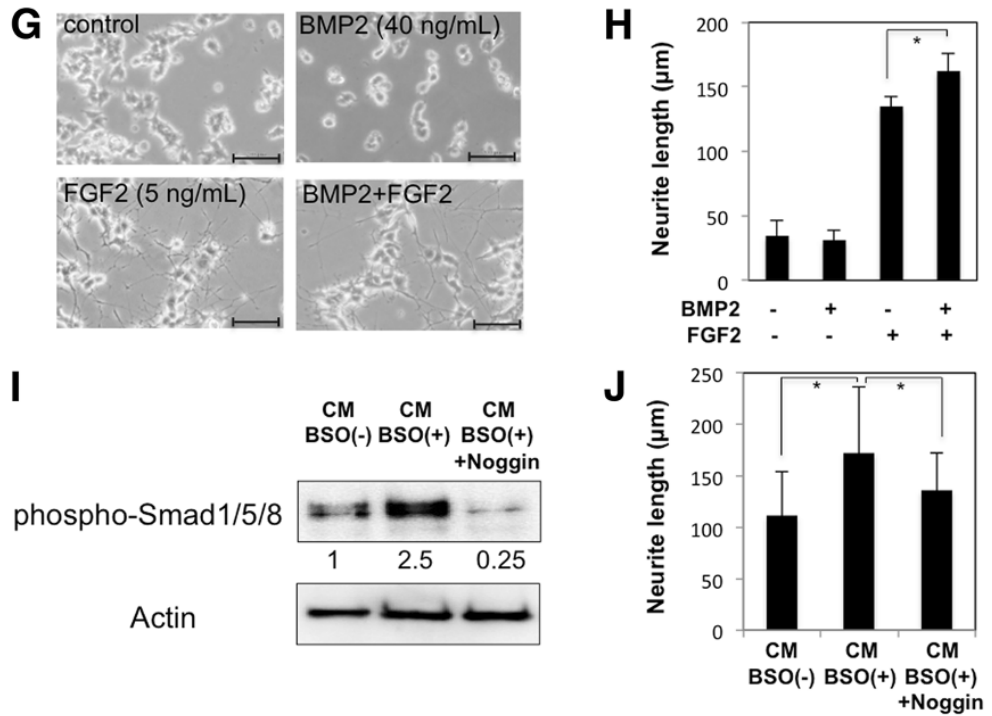

Figure 3 Transcription and secretion of BMP2 and FGF2 were increased in hADMPCs exposed to oxidative stress. (A, B) Upregulation of BMP2 (A) and FGF2 (B) mRNA in hADMPCs by BSO in a dose-dependent manner. (C, D) Secretion of BMP2 (C) and FGF2 (D) from hADMPCs in medium alone (cont) or with addition of $1 \mathrm{mM} \mathrm{BSO}(\mathrm{BSO})$ was analyzed by ELISA. (E, F) NAC treatment repressed the expression levels of BMP2 and FGF2 upregulated by BSO to the control levels. Expression of BMP2 (E) and FGF2 (F) mRNA was analyzed by q-PCR. CDNA was generated from total RNA extracted from hADMPCs (cont), hADMPCs treated with $1 \mathrm{mM} \mathrm{BSO}$ (BSO), $1 \mathrm{mM}$ BSO + $5 \mathrm{mM} \mathrm{NAC}$ (BSO + NAC), and $5 \mathrm{mM} \mathrm{NAC} \mathrm{(NAC).}$ The most reliable internal control gene was determined using the geNorm Software. (G, H) PC12 cells were cultured in differentiation medium alone (control), or differentiation medium supplemented with BMP2 (40 ng/mL), FGF2 (5 ng/mL), or both BMP2 and FGF2 (BMP2 + FGF2) for 2 days. (G) Representative images of neurite outgrowth in PC12 cells. Scale bars, $200 \mu \mathrm{m}$. (H) One hundred individual neurites were measured in each sample using Dynamic Cell Count Analyzer BZ-H1C (Keyence) and average neurite length was calculated. *, P $<0.05$ (Student's t test). (I, J) PC12 cells were cultured in CM-BSO (-), CM-BSO (+), or CM-BSO (+) added with recombinant murine Noggin (200 ng/mL). (I) Western blot analysis of PC12 cells $1 \mathrm{~h}$ after CM treatment. Proteins extracted from each sample were resolved by SDS-PAGE, transferred to a membrane, and probed with anti-phosphorylated Smad1/5/8 (phospho-Smad1/5/8) and anti-Actin. Numbers below blots indicate relative band intensities as determined by the ImageJ software. (J) Two days after CM treatment, 100 individual neurites in PC12 cells were measured in each sample using Dynamic Cell Count Analyzer BZ-H1C (Keyence) and average neurite length was calculated. ${ }^{*}, \mathrm{P}<0.05$ (Student's $\mathrm{t}$ test). 
previously been shown to induce neurite outgrowth in PC12 cells [26,27], we examined the effect of BMP2 and FGF2 on neurite outgrowth. We confirmed that PC12 cells did not differentiate effectively by BMP2 treatment alone, but BMP2 significantly augmented FGF2-induced neurite outgrowth in PC12 cells (Figure 3G and $\mathrm{H}$ ), as previously reported. Moreover, in order to confirm the effect of BMP2 on neurite outgrowth in PC12 cells, $200 \mathrm{ng} /$ $\mathrm{mL}$ of Noggin, an antagonist of BMP signaling, was added to $\mathrm{CM}-\mathrm{BSO}(+)$. Addition of Noggin significantly suppressed the CM-BSO (+)-evoked phosphorylation of Smad1/5/8 (Figure 3I) and shortened the length of neurite outgrowth in PC12 cells (Figure 3J).

To address the question of which intracellular signaling pathways are affected by oxidative stress in
hADMPCs, we focused on MAPK signaling since previous studies had suggested that accumulation of ROS in cells led to the activation of Erk1/2, p38, and JNK MAPK [28,29]. Western blotting revealed that BSO treatment markedly activated the p38 MAPK pathway; SB203580 could inhibit the activation, and U0126 treatment stimulated the activation (Figure 4A). ERK1/2 MAPK was significantly phosphorylated by BSO treatment, and ERK1/2 activation was reduced to the control level by treatment with U0126 (Figure 4B). In contrast, JNK activation was not observed in BSO-treated hADMPCs (Figure 4B). Therefore, we further investigated the relationship between increases in BMP2 and FGF2 expression and activation of the p38 and ERK1/2 MAPK signaling pathways by oxidative stress. Treatment

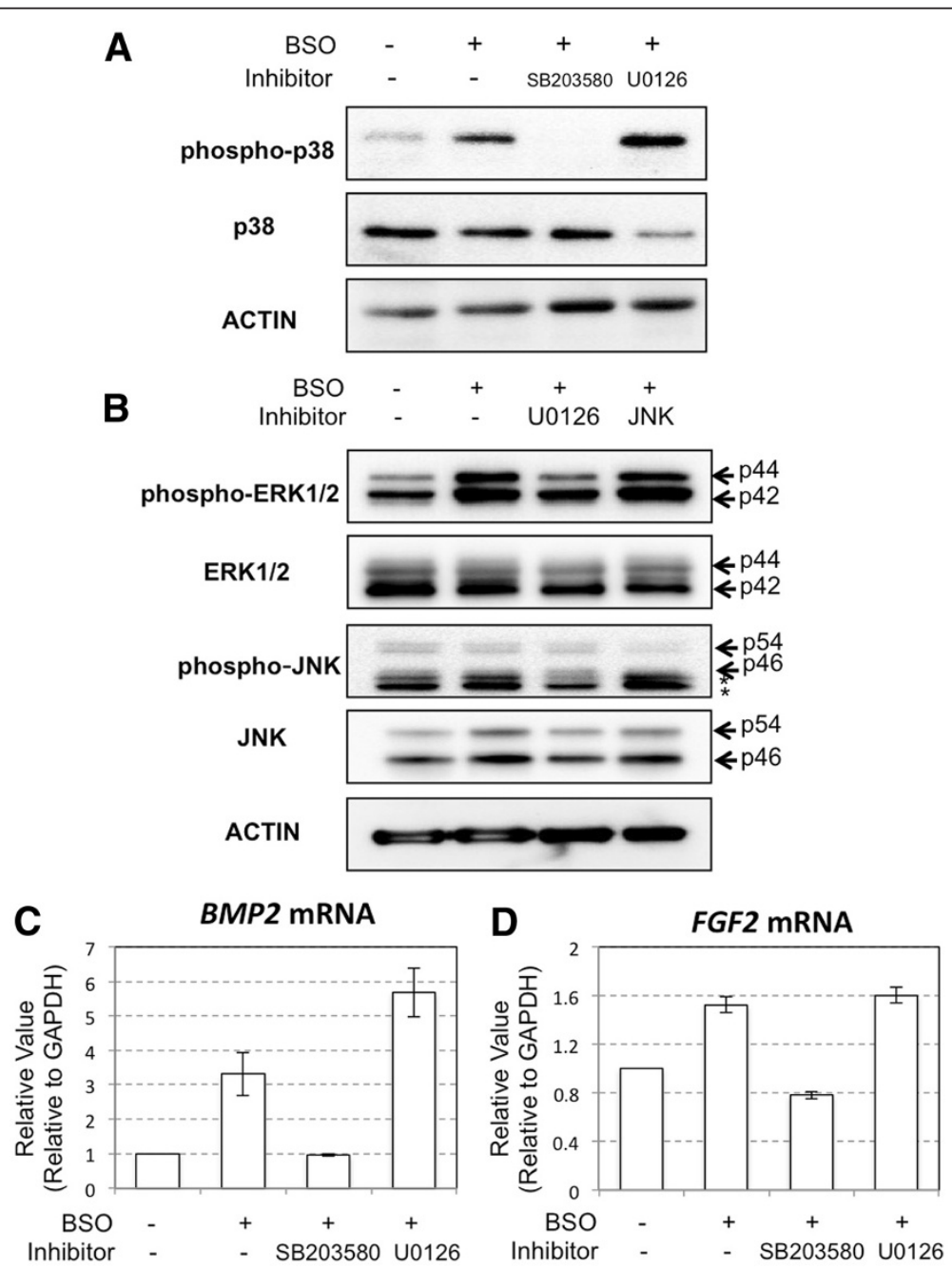

Figure 4 BMP2 and FGF2 were upregulated through activation of p38 MAPK. Inhibiton of p38 MAPK resulted in the supression of BMP2 and FGF2 transcripts upregulated by BSO treatment in hADMPCs. hADMPCs were pre-treated with $10 \mu \mathrm{M}$ of SB203580, $10 \mu \mathrm{M}$ of $\mathrm{U} 0126$ or $10 \mu \mathrm{M}$ of JNK inhibitor II for $2 \mathrm{~h}$ followed by $1 \mathrm{mM} \mathrm{BSO}$ treatment for $16 \mathrm{~h}$. The medium was replaced with fresh culture medium and the cells were cultured for another 2 days. (A) Western blot analysis of p38 MAPK activation in hADMPCs. (B) Western blot analysis of ERK1/2 MAPK, JNK SAPK activation in hADMPCs. (C, D) Transcription levels of BMP2 (C) and FGF2 (D) were analyzed by q-PCR. The most reliable internal control gene was determined using the geNorm Software. 
with the p38 MAPK inhibitor SB203580 dramatically downregulated the expression levels of BMP2 and FGF2 to control levels (Figure $4 \mathrm{C}$ and $\mathrm{D}$ ). In contrast, the Erk1/2 MAPK inhibitor U0126 had no effect on FGF2 expression levels and led to a slight increase in $B M P 2$ expression (Figure 4C and D).

\section{MKK6-mediated activation of p38 MAPK increases BMP2} and FGF2 expression in hADMPCs

To further confirm the involvement of p38 MAPK in the regulation of BMP2 and FGF2, hADMPCs were transduced with a lentiviral vector expressing constitutively active MKK6 (MKK6 (glu)) [30] from an EF1 $\alpha$ promoter. As shown in Figure 5A, lentiviral transduction of MKK6 (glu) led to expression of Flag-tagged MKK6 (glu) in hADMPCs. Moreover, the expression of MKK6 (glu) resulted in activation of p38 MAPK as expected [30] (Figure 5A), and upregulation of BMP2 and FGF2 expression (Figure 5B-E).

\section{NF-KB is not activated in hADMPCs exposed to oxidative stress}

It has been reported that NF- $\mathrm{KB}$ directly binds to the $B M P 2$ promoter to induce its expression [31], and MSK1, a downstream molecule of p38 MAPK, is involved in NF- $\mathrm{KB}$ transactivation [32]. Therefore, we



Figure 5 Activation of p38 MAPK by a constitutively active form of MKK6 resulted in elevated expression of BMP2 and FGF2. (A) A lentiviral vector expressing Flag-tagged MKK6 (glu) was transfected into hADMPCs. Expression of Flag-tagged MKK6 (glu), phosphorylated p38 MAPK and p38 MAPK was analyzed by western blotting. A CSII-EF-EGFP lentiviral vector was infected as a control (GFP). Actin was detected as an internal control. (B, C) Transcriptional levels of BMP2 (B) and FGF2 (C) were analyzed by q-PCR. The most reliable internal control gene was determined using the geNorm Software. (D, E) BMP2 (D) and FGF2 (E) secretion was analyzed by ELISA. 
hypothesized that p38 MAPK-mediated activation of NF- $\mathrm{KB}$ might contribute to elevated expression of $B M P 2$ mRNA. To confirm this hypothesis, transcriptional activation of NF- $\mathrm{kB}$ was examined by measuring luciferase activity driven by the synthetic NF- $\mathrm{kB}$ response element. We found that transcriptional activity of NF-kB was not stimulated by BSO treatment (Figure 6A), and immunocytochemical analysis also revealed that NF- $\mathrm{kB}$ was not activated (nuclear localization of NF-kB/p65 was rarely observed) in BSO-treated hADMPCs (Figure 6B). These results suggested that elevated expression of $B M P 2$ mRNA is not mediated by NF- $\mathrm{kB}$ signaling.

Our current data thus demonstrate the crucial role of ROS, via activation of the p38 MAPK signaling pathway, in regulating expression levels of the neurotrophic factors BMP2 and FGF2 in hADMPCs. The overall model that we propose, based upon our findings, is shown in Figure 7.

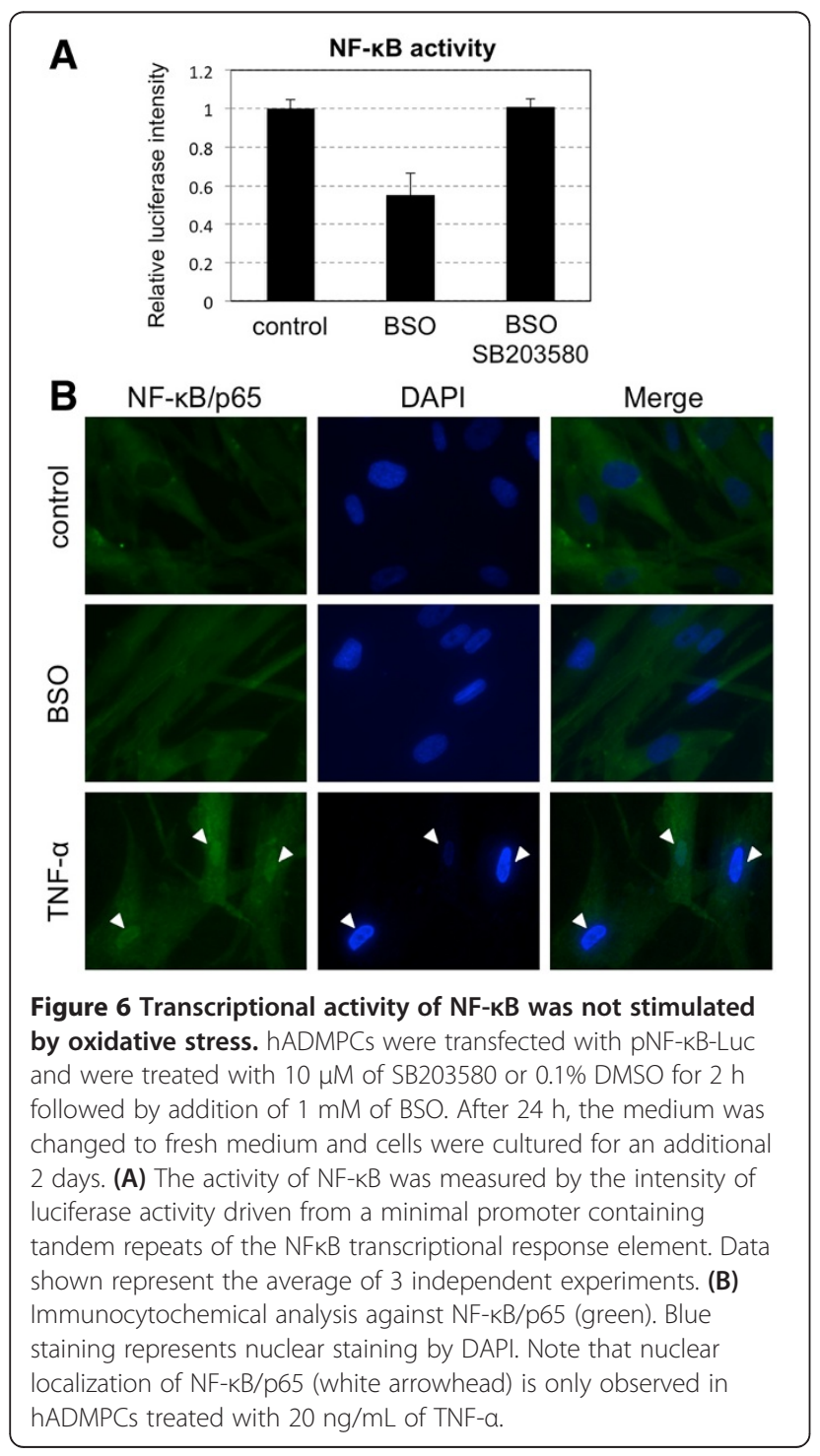

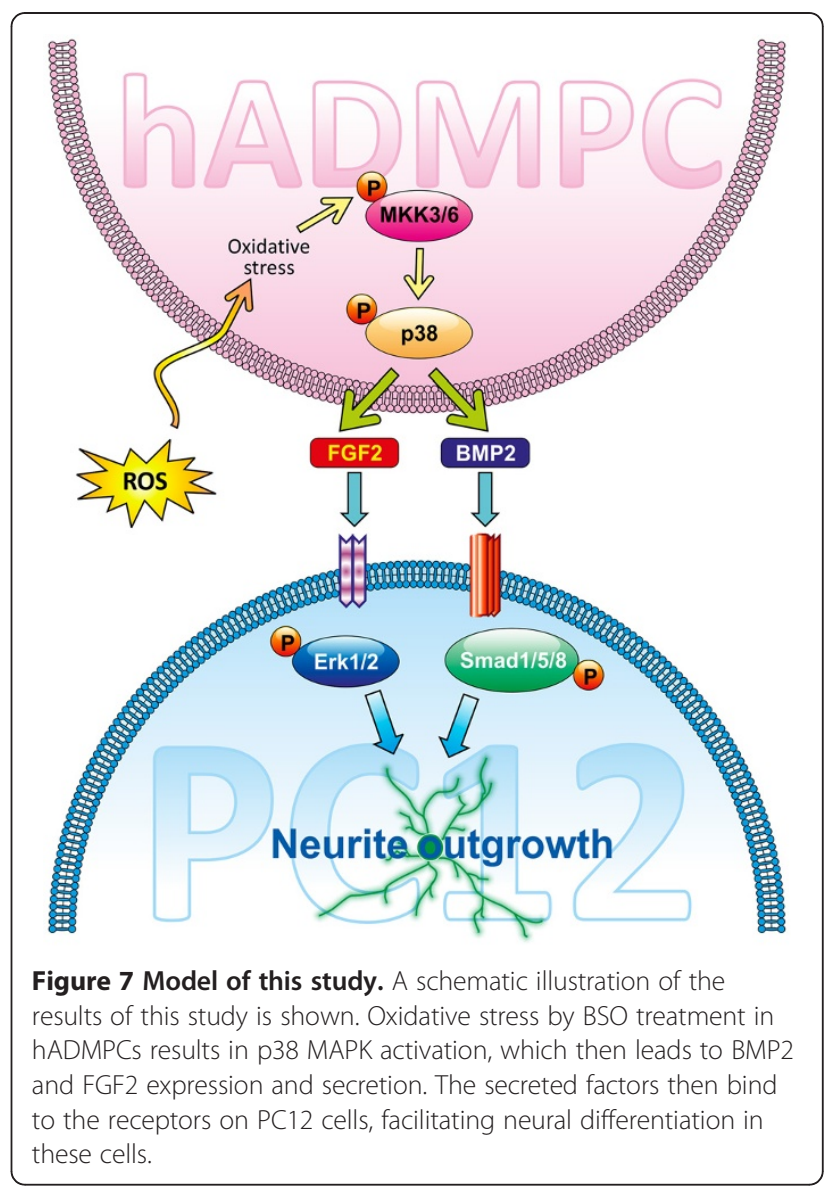

\section{Discussion}

In this study, we investigated the effect of oxidative stress in hADMPCs on the induction of neuronal differentiation. Such mechanisms may explain how administration of hADMPCs to neurodegenerative lesions enhances endogenous repair mechanisms via neurogenesis of endogenous neural progenitor and stem cells. Damaged tissues, such as the brain tissue of patients who have suffered from ischemic stroke, are subject to inflammation and the generation of reactive oxygen species (ROS) [17,18]. Our data demonstrated that hADMPCs, when exposed to oxidative stress, facilitate neuronal differentiation in rat pheochromocytoma cell line PC12 cells by upregulation of fibroblast growth factor 2 (FGF2) and bone morphogenetic protein 2 (BMP2) secretion through p38 MAPK activation.

Our results show that BMP2 and FGF2 were upregulated in hADMPCs when exposed to buthionine sulfoximine (BSO), a glutathione-synthesis inhibitor that leads to oxidative stress. These findings may have therapeutic implications in neurodegenerative diseases. We concluded that BMP2 and FGF2 secreted from hADMPCs that had been exposed to oxidative stress were the main inducers of neurite outgrowth in PC12 cells. Erk1/2 and 
Smad1/5/8 were significantly activated in these cells (Figure 2), while other growth factors known to induce neurite outgrowth in PC12 cells such as nerve growth factor (NGF) and vascular endothelial growth factor (VEGF) were not observed to be upregulated by BSO treatment (data not shown). We confirmed that BMP2 enhanced the effect that FGF2 had on the differentiation of PC12 cells (Figure 3), supporting our idea that hADMPCs under oxidative stress conditions secrete BMP2 and FGF2 and that this contributes to neuronal differentiation. Consistent with our conclusions, it has been reported that BMP2, via activation of a Smad signaling pathway, facilitated FGF2-induced neuronal differentiation in PC12 cells [26,27]. However, since hADMPCs have been reported to secrete many growth factor including NGF, VEGF, HGF, and IGF [11,15,33], we cannot exclude the possibility that BMP2 and FGF2 are acting cooperatively with these growth factors to facilitate neurite outgrowth in PC12 cells. Thus, the precise molecular mechanisms of induction of PC12 differentiation and the precise expression profiles in BSO-treated hADMPCs need to be further investigated.

Recently, BMP signaling through Smad1/5/8 has been reported to contribute to neurite outgrowth in dorsal root ganglion neurons both in vitro and in vivo [34,35]. Moreover, BMP2 has been shown to have neurotrophic effects on midbrain dopaminergic neurons [36], ventral mesencephalic neurons [37], mouse embryonic striatal neurons [38], and nitrergic and catecholaminergic enteric neurons [39]. Moreover, FGF2 is trophic for neurons, glias, and endothelial cells in the central nervous system. FGF2 also prevents downregulation of the antiapoptotic protein Bcl-2 in ischemic brain tissue and limits excitotoxic damage to the brain through an activindependent mechanism [40]. These findings are consistent with our hypothesis that hADMPCs secret BMP2 and FGF2 to induce neurogenesis in neurodegenerative lesions in response to oxidative stress.

As it has been shown that ROS activate ERKs, JNKs, and p38 MAPKs $[28,29]$, we examined the MAPK signaling pathway in hADMPCs exposed to oxidative stress and found that BSO treatment resulted in significant activation of ERK1/2 and p38 MAPK. Intriguingly, addition of SB203580, a specific inhibitor of p38 MAPK, but not the ERK inhibitor U0126, suppressed BMP2 and FGF2 expression in BSO-treated hADMPCs to control levels (Figure 4), suggesting that p38 MAPK was contributing to upregulation of BMP2 and FGF2 in hADMPCs when exposed to oxidative stress. Moreover, lentiviral transduction of the constitutively active form of MKK6, a MAPKK that selectively activates p38 MAPK isoforms [30], resulted in upregulation of BMP2 and FGF2 and this also demonstrated the crucial role of the p38 MAPK cascade in the regulation of BMP2 and FGF2. In primary human endothelial cells, p38-dependent regulation of BMP2 expression was reported previously. Viemann et al. [41] investigated the genes that were induced by inflammatory stimulation with tumor necrosis factor $\alpha$ (TNF- $\alpha$ ) and classified these genes into 2 categories based on whether they were regulated in an NF-kB-dependent or p38 MAPK-dependent manner. Consistent with our findings, they found that significant induction of BMP2 expression by TNF- $\alpha$ was markedly suppressed by SB202190, an inhibitor of p38 MAPK. These results support the hypothesis that activation of the p38 MAPK pathway in hADMPCs in response to inflammation surrounding neurodegenerative lesions leads to induction of BMP2 and FGF2, which in turn support regeneration of neuronal cells.

It has been known that NF- $\kappa B$ directly binds to the BMP2 promoter to induce its expression [31], and MSK1, a downstream molecule of p38 MAPK, is involved in NF- $\mathrm{kB}$ transactivation [32]. However, we did not observe an elevation of NF- $\mathrm{kB}$ transcriptional activity in hADMPCs when they were exposed to oxidative stress (Figure 6). The mechanism of p38-dependent regulation of gene expression is not completely understood, and the precise mechanism by which p38 MAPK regulates the expression of $\mathrm{BMP} 2$ and FGF2 remains to be determined.

In this study, we also found that suppression of ERK1/2 MAPK by U0126 in BSO-treated hADMPCs resulted in slight activation of p38 MAPK (Figure 4A). Consistent with this, the expression level of $B M P 2$ mRNA was also upregulated when cells exposed to oxidative stress were pretreated with U0126 (Figure 4C). Previously, "seesaw cross-talk" between ERK and p38 MAPK signaling has been reported; i.e., the MEK inhibitor caused a decrease in the phosphorylation level of ERK and an increase in that of p38, whereas the p38 inhibitor had the opposite effect [42-44]. We did not investigate the phosphorylation of ERK1/2 in SB203580-treated hADMPCs, but it may be possible that seesaw cross-talk also occurs in our system.

\section{Conclusions}

In summary, the results obtained in this study have demonstrated the potential use of hADMPCs for the treatment of neurodegenerative diseases such as ischemic stroke, Parkinson's disease, Alzheimer's disease, and spinal cord injury, in which the transplanted hADMPCs might be exposed to oxidative stress. Moreover, the p38dependent modulation of BMP2 and FGF2 expression observed in this study is expected to be a new therapeutic target for neurodegenerative disorders.

\section{Materials and methods \\ Adipose tissue samples}

Subcutaneous adipose tissue samples (10-50 g, each) were resected during plastic surgery in 5 females (age, 
20-60 years) as excess discards. The study protocol was approved by the Review Board for Human Research of Kobe University Graduate School of Medicine, Foundation for Biomedical Research and Innovation and Kinki University Pharmaceutical Research and Technology Institute (reference number: 10-005). Each subject provided a signed informed consent.

\section{Cell culture}

PC12 cells were obtained from the Health Science Research Resources Bank (Osaka, Japan) and maintained in RPMI1640 media supplemented with 10\% heat-inactivated horse serum and 5\% fetal bovine serum. For differentiation, the cells were plated in 6-well culture plates coated with collagen type I (Nitta Gelatin, Osaka, Japan) and the medium was replaced with differentiation medium (RPMI1640 supplemented with 1\% horse serum and 0.5\% fetal bovine serum) or conditioned medium from hADMPCs. NGF (50 ng/mL), BMP2 (40 ng/mL) or FGF2 ( $5 \mathrm{ng} / \mathrm{mL}$ ) were added to the differentiation medium. Recombinant murine Noggin (200 ng/mL: PeproTech, NJ, USA) was added to conditioned medium from BSOtreated hADMPCs. hADMPCs were isolated as previously reported [4-6,45,46] and maintained in a medium containing 60\% DMEM-low glucose, 40\% MCDB-201 medium (Sigma Aldrich, St. Louis, MO, USA), 1× insulintransferrin-selenium (Gibco Invitrogen, NY, USA), $1 \mathrm{nM}$ dexamethasone (Sigma Aldrich), $100 \mathrm{mM}$ ascorbic acid 2phosphate (Wako, Osaka, Japan), $10 \mathrm{ng} / \mathrm{mL}$ epidermal growth factor (PeproTech), and 5\% fetal bovine serum. The cells were plated to a density of $5 \times 10^{3}$ cells $/ \mathrm{cm}^{2}$ on fibronectin-coated dishes, and the medium was replaced every 2 days.

\section{Preparation of conditioned medium from hADMPCs}

Two days after plating, hADMPCs were treated with BSO (concentrations used were varied in each experiment and are indicated in the results and figure legends) for $16 \mathrm{~h}$. The medium was replaced with fresh culture medium for 2 days followed by replacement with PC12 cell differentiation medium. After 2 more days, the medium was removed for use as conditioned medium. For preparation of the conditioned medium from hADMPCs in which one of the three, p38, Erk1/2, or JNK MAPK, was inhibited, hADMPCs were pretreated with $10 \mu \mathrm{M}$ SB203580 (Promega, WI, USA), $10 \mu \mathrm{M}$ U0126 (Promega), or $10 \mu \mathrm{M}$ JNK inhibitor II (EMD4 Bioscience, CA, USA), respectively, for $2 \mathrm{~h}$ and subsequently treated with $1 \mathrm{mM}$ BSO.

\section{Measurement of GSH/GSSG ratio}

Ratios of reduced glutathione (GSH) to oxidized glutathione (GSSG) were measured using the GSH/GSSG-Glo assay kit (Promega) following the manufacturer's protocol.

\section{Measurement of reactive oxygen species production}

Cells were harvested and incubated with $10 \mu \mathrm{M}$ 5-(and6)-chloromethyl-2',7'-dichlorodihydrofluorescein diacetate, acetyl ester (CM- $\left.\mathrm{H}_{2} \mathrm{DCFDA}\right)$. The amount of intracellular ROS production was proportional to green fluorescence, as analyzed with a Guava easyCyte $8 \mathrm{HT}$ flow cytometer (Millipore) using an argon laser at $488 \mathrm{~nm}$ and a 525/30 $\mathrm{nm}$ band pass filter, and dead cells were excluded with the LIVE/DEAD fixable far red dead cell stain kit (Invitrogen).

\section{Western blot analysis}

Cells were washed with ice-cold phosphate-buffered saline and lysed with M-PER Mammalian Protein Extraction Reagent (Thermo Scientific Pierce, IL, USA) following the manufacturer's instructions. Equal amounts of proteins were separated by sodium dodecylsulfate polyacrylamide gel electrophoresis (SDS-PAGE), transferred to polyvinylidene fluoride (PVDF) membranes (Immobilon-P; Millipore, MA, USA), and probed with antibodies against phospho-Erk1/2 (\#4370), Erk1/2 (\#4695), phospho-38 (\#9215), p38 (\#9212), phosphoSmad1/5/8 (\#9511), phospho-Akt (\#4060), Akt (\#4691), phospho-JNK (\#9251), JNK (\#9258) (all from Cell Signaling Technology, MA, USA) and actin (Millipore). Horseradish peroxidase (HRP)-conjugated anti-rabbit and anti-mouse secondary antibodies (Cell Signaling Technology, Danvers, MA, USA) were used as probes and immunoreactive bands were visualized with the Immobilon Western Chemiluminescent HRP substrate (Millipore). The band intensity was measured using ImageJ software.

\section{RNA extraction, CDNA generation, and quantitative polymerase chain reaction (q-PCR)}

Total RNA was extracted using the RNeasy Mini Kit (Qiagen, Hilden, Germany) following the manufacturer's instructions. cDNA was generated from $1 \mu \mathrm{g}$ of total RNA using the Verso cDNA Synthesis Kit (Thermo Scientific) and purified with the MinElute PCR Purification Kit (Qiagen). Q-PCR analysis was carried out using the SsoFast EvaGreen supermix (Bio-Rad, CA, USA) according to the manufacturer's protocols. The relative expression value of each gene was calculated using a $\Delta \Delta \mathrm{Ct}$ method and the most reliable internal control gene was determined using the geNorm Software (http://medgen.ugent.be/ jvdesomp/genorm/). Details of the primers used in these experiments are available on request.

\section{Enzyme-linked immunosorbent assay}

Enzyme-linked immunosorbent assay (ELISA) was performed using the Quantikine BMP-2 Immunoassay System and Quantikine FGF-2 Immunoassay System (R\&D 
Systems, MN, USA) following the manufacturer's protocols.

\section{Plasmid construction and lentivirus production}

Flag-tagged MKK6 (glu) [30] was provided by Addgene (pcDNA3-Flag MKK6 (glu); Addgene plasmid 13518). Flag-tagged MKK6 (glu) was cloned into a pENTR11 vector (Invitrogen). An iresGFP fragment was subsequently cloned into the plasmid to produce the entry vector pENTR11-MKK6 (glu)-iresGFP. The entry vector and CSII-EF-RfA (kindly provided by Dr. Miyoshi, RIKEN BioResource Center, Tsukuba, Japan) were incubated with LR clonase II enzyme mix (Invitrogen) to generate CSII-EF-MKK6 (glu)-iresGFP. The resultant plasmid was mixed with packaging plasmids (pCAG$\mathrm{HIVg} / \mathrm{p}$ and $\mathrm{pCMV}-\mathrm{VSVG}-\mathrm{RSV}-\mathrm{Rev}$, kindly provided by Dr. Miyoshi) and transfected into $293 \mathrm{~T}$ cells. The supernatant medium, which contained lentiviral vectors, was collected 2 days after transduction and concentrated by centrifugation $\left(6000 \mathrm{G}, 15 \mathrm{~h}, 4^{\circ} \mathrm{C}\right)$.

\section{Luciferase assay}

hADMPCs were transfected with pGL4.74 (Promega) and either pTAL-Luc or pNF-kB-Luc by TransIT-2020 (TaKaRa-Bio). The cells were then treated with $10 \mu \mathrm{M}$ of SB203580 or $0.1 \%$ DMSO for $2 \mathrm{~h}$ followed by addition of $1 \mathrm{mM}$ of BSO. After $24 \mathrm{~h}$, the medium was changed to fresh medium and cells were cultured for an additional 2 days. The activity of NF- $\mathrm{kB}$ was measured using the Dual Luciferases Assay System (Promega) according to the manufacturer's protocol.

\section{Immunocytochemistry}

hADMPCs were fixed with $4 \%$ paraformaldehyde in PBS for $10 \mathrm{~min}$ at $4^{\circ} \mathrm{C}$ and then washed 3 times in PBS. Blocking was performed with PBSMT (PBS containing $0.1 \%$ Triton X-100, $2 \%$ Skim Milk) for $1 \mathrm{~h}$ at room temperature. The cells were then incubated with rabbit monoclonal antibody against NF-kB p65 (Cell Signaling; \#8242; 1/100 dilution) overnight at $4^{\circ} \mathrm{C}$. After washing with PBS, cells were incubated with Alexa 488 conjugated anti-rabbit IgG (Invitrogen; 1/1000 dilution) for $1 \mathrm{~h}$. The cells were counterstained with 4'-6-diamidino2-phenylindole (DAPI) (Invitrogen) to identify cellular nuclei.

\section{Competing interests}

None of the authors have any competing interests related to the manuscript.

\section{Authors' contributions}

MM carried out the FACS analysis, QPCR analysis, ELISA, immunofluorescent staining, and cell culture, participated in the study design, and drafted the manuscript. HM participated in the study design, carried out the western blot analysis, luciferase assay, and cell culture, and drafted the manuscript. $\mathrm{AU}$ carried out western blot analysis, constructed the plasmids, and generated the lentiviral vectors. YN carried out QPCR analysis and performed the statistical analysis. Al resected subcutaneous adipose tissue samples during plastic surgery. $\mathrm{HO}$ and AM isolated hADMPCs from human adipose tissues. TH conceived the study, participated in its design and coordination, and helped to draft the manuscript. All authors read and approved the final manuscript.

\section{Acknowledgements}

We thank A Nishikawa, T Fukase, T Sasaki, T Shoji, K Nakagita, S Fukui, and K Honjo for technical support. We thank Dr. Roger Davis for providing the pcDNA3-Flag MKK6 (glu) plasmid and Dr. Hiroyuki Miyoshi for the CSII-EFRfA, pCMV-VSVG-RSV-Rev and pCMV-HIVg/p plasmids. This work was supported in part by grants from the Ministry of Health, Labor, and Welfare of Japan and a grant from the Program for Promotion of Fundamental Studies in Health Sciences of the National Institute of Biomedical Innovation (NIBIO).

\section{Author details}

${ }^{1}$ Pharmaceutical Research and Technology Institute, Kinki University, 3-4-1 Kowakae, Higashi-Osaka, Osaka 577-8502, Japan. ${ }^{2}$ Department of Somatic Stem Cell Therapy and Health Policy, Foundation for Biomedical Research and Innovation, TRI305, 1-5-4 Minatojima-minamimachi, Chuo-ku, Kobe, Hyogo 650-0047, Japan. ${ }^{3}$ Department of Plastic Surgery, Kobe University Hospital, Kobe, Japan.

Received: 28 March 2012 Accepted: 2 August 2012

Published: 7 August 2012

\section{References}

1. Pittenger MF, Mackay AM, Beck SC, Jaiswal RK, Douglas R, Mosca JD, Moorman MA, Simonetti DW, Craig S, Marshak DR: Multilineage potential of adult human mesenchymal stem cells. Science 1999, 284:143-147.

2. Bieback K, Kern S, Kluter H, Eichler H: Critical parameters for the isolation of mesenchymal stem cells from umbilical cord blood. Stem Cells 2004, 22:625-634.

3. Zuk PA, Zhu M, Ashjian P, De Ugarte DA, Huang Jl, Mizuno H, Alfonso ZC, Fraser JK, Benhaim P, Hedrick MH: Human adipose tissue is a source of multipotent stem cells. Mol Biol Cell 2002, 13:4279-4295.

4. Okura H, Komoda H, Saga A, Kakuta-Yamamoto A, Hamada Y, Fumimoto $Y$, Lee CM, Ichinose A, Sawa Y, Matsuyama A: Properties of hepatocyte-like cell clusters from human adipose tissue-derived mesenchymal stem cells. Tissue Eng Part C Methods 2010, 16:761-770.

5. Okura H, Matsuyama A, Lee CM, Saga A, Kakuta-Yamamoto A, Nagao A, Sougawa N, Sekiya N, Takekita K, Shudo Y, et al: Cardiomyoblast-like cells differentiated from human adipose tissue-derived mesenchymal stem cells improve left ventricular dysfunction and survival in a rat myocardial infarction model. Tissue Eng Part C Methods 2010, 16:417-425.

6. Komoda H, Okura H, Lee CM, Sougawa N, Iwayama T, Hashikawa T, Saga A, Yamamoto-Kakuta A, Ichinose A, Murakami S, Sawa Y, Matsuyama A: Reduction of $\mathrm{N}$-glycolylneuraminic acid xenoantigen on human adipose tissue-derived stromal cells/mesenchymal stem cells leads to safer and more useful cell sources for various stem cell therapies. Tissue Eng Part A 2010, 16:1143-1155.

7. Okura H, Komoda H, Fumimoto $Y$, Lee CM, Nishida T, Sawa Y, Matsuyama A: Transdifferentiation of human adipose tissue-derived stromal cells into insulin-producing clusters. J Artif Organs 2009, 12:123-130.

8. Safford KM, Safford SD, Gimble JM, Shetty AK, Rice HE: Characterization of neuronal/glial differentiation of murine adipose-derived adult stromal cells. Exp Neurol 2004, 187:319-328.

9. Leu S, Lin YC, Yuen CM, Yen CH, Kao YH, Sun CK, Yip HK: Adipose-derived mesenchymal stem cells markedly attenuate brain infarct size and improve neurological function in rats. J Trans/ Med 2010, 8:63.

10. Ikegame Y, Yamashita K, Hayashi S, Mizuno H, Tawada M, You F, Yamada K, Tanaka Y, Egashira Y, Nakashima S, Yoshimura S, Iwama T: Comparison of mesenchymal stem cells from adipose tissue and bone marrow for ischemic stroke therapy. Cytotherapy 2011, 13:675-685.

11. Tan B, Luan Z, Wei X, He Y, Wei G, Johnstone BH, Farlow M, Du Y. AMPactivated kinase mediates adipose stem cell-stimulated neuritogenesis of PC12 cells. Neuroscience 2011, 181:40-47.

12. Reid AJ, Sun M, Wiberg M, Downes S: Terenghi G. Kingham PJ: Nerve repair with adipose-derived stem cells protects dorsal root ganglia neurons from apoptosis. Neuroscience; 2011. 
13. Rehman J, Traktuev D, Li J, Merfeld-Clauss S, Temm-Grove CJ, Bovenkerk JE, Pell CL, Johnstone BH, Considine RV, March KL: Secretion of angiogenic and antiapoptotic factors by human adipose stromal cells. Circulation 2004, 109:1292-1298.

14. Lee EY, Xia Y, Kim WS, Kim MH, Kim TH, Kim KJ, Park BS, Sung JH: Hypoxiaenhanced wound-healing function of adipose-derived stem cells: increase in stem cell proliferation and up-regulation of VEGF and bFGF. Wound Repair Regen 2009, 17:540-547.

15. Lu S, Lu C, Han Q, Li J, Du Z, Liao L, Zhao RC: Adipose-derived mesenchymal stem cells protect PC12 cells from glutamate excitotoxicity-induced apoptosis by upregulation of XIAP through PI3-K/ Akt activation. Toxicology 2011, 279:189-195.

16. McCoy MK, Martinez TN, Ruhn KA, Wrage PC, Keefer EW, Botterman BR, Tansey KE, Tansey MG: Autologous transplants of Adipose-Derived Adult Stromal (ADAS) cells afford dopaminergic neuroprotection in a model of Parkinson's disease. Exp Neurol 2008, 210:14-29.

17. Flamm ES, Demopoulos HB, Seligman ML, Poser RG, Ransohoff J: Free radicals in cerebral ischemia. Stroke 1978, 9:445-447.

18. Alexandrova ML, Bochev PG: Oxidative stress during the chronic phase after stroke. Free Radic Biol Med 2005, 39:297-316.

19. Lambeth JD: NOX enzymes and the biology of reactive oxygen. Nat Rev Immunol 2004, 4:181-189.

20. Simpson JE, Ince PG, Haynes $\sqcup$, Theaker $R$, Gelsthorpe $C$, Baxter L, Forster $G$, Lace GL, Shaw PJ, Matthews FE, Savva GM, Brayne C, Wharton SB, MRC Cognitive Function and Ageing Neuropathology Study Group: Population variation in oxidative stress and astrocyte DNA damage in relation to Alzheimer-type pathology in the ageing brain. Neuropathol Appl Neurobiol 2010, 36:25-40.

21. Cai Z, Zhao B, Ratka A: Oxidative Stress and beta-Amyloid Protein in Alzheimer's Disease. Neuromolecular Med 2011, 13:223-250.

22. Beal MF: Mitochondria, oxidative damage, and inflammation in Parkinson's disease. Ann N Y Acad Sci 2003, 991:120-131.

23. Henchcliffe C, Beal MF: Mitochondrial biology and oxidative stress in Parkinson disease pathogenesis. Nat Clin Pract Neurol 2008, 4:600-609.

24. Minghetti L, Ajmone-Cat MA, De Berardinis MA, De Simone R: Microglial activation in chronic neurodegenerative diseases: roles of apoptotic neurons and chronic stimulation. Brain Res Brain Res Rev 2005, 48:251-256.

25. Colton CA, Chernyshev ON, Gilbert DL, Vitek MP: Microglial contribution to oxidative stress in Alzheimer's disease. Ann N Y Acad Sci 2000, 899:292-307.

26. Hayashi H, Ishisaki A, Suzuki M, Imamura T: BMP-2 augments FGF-induced differentiation of PC12 cells through upregulation of FGF receptor-1 expression. J Cell Sci 2001, 114:1387-1395.

27. Hayashi H, Ishisaki A, Imamura T: Smad mediates BMP-2-induced upregulation of FGF-evoked PC12 cell differentiation. FEBS Lett 2003, 536:30-34.

28. Son Y, Cheong YK, Kim NH, Chung HT, Kang DG, Pae HO: MitogenActivated Protein Kinases and Reactive Oxygen Species: How Can ROS Activate MAPK Pathways? J Signal Transduct 2011, 2011:792639.

29. Ito K, Hirao A, Arai F, Takubo K, Matsuoka S, Miyamoto K, Ohmura M, Naka K, Hosokawa K, Ikeda Y, Suda T: Reactive oxygen species act through p38 MAPK to limit the lifespan of hematopoietic stem cells. Nat Med 2006 12:446-451.

30. Raingeaud J, Whitmarsh AJ, Barrett T, Derijard B, Davis RJ: MKK3- and MKK6-regulated gene expression is mediated by the p38 mitogenactivated protein kinase signal transduction pathway. Mol Cell Biol 1996, 16:1247-1255.

31. Feng JQ, Xing $L$, Zhang JH, Zhao M, Horn D, Chan J, Boyce BF, Harris SE, Mundy GR, Chen D: NF-kappaB specifically activates BMP-2 gene expression in growth plate chondrocytes in vivo and in a chondrocyte cell line in vitro. J Biol Chem 2003, 278:29130-29135.

32. Vermeulen L, De Wilde G, Van Damme P: Vanden Berghe W, Haegeman G: Transcriptional activation of the NF-kappaB p65 subunit by mitogenand stress-activated protein kinase-1 (MSK1). EMBO J 2003, 22:1313-1324.

33. Rasmussen JG, Frobert O, Pilgaard L, Kastrup J, Simonsen U, Zachar V, Fink T: Prolonged hypoxic culture and trypsinization increase the proangiogenic potential of human adipose tissue-derived stem cells. Cytotherapy 2011, 13:318-328.

34. Parikh P, Hao Y, Hosseinkhani M, Patil SB, Huntley GW, Tessier-Lavigne M, Zou H: Regeneration of axons in injured spinal cord by activation of bone morphogenetic protein/Smad1 signaling pathway in adult neurons. Proc Natl Acad Sci U S A 2011, 108:E99-E107.

35. Ma CH, Brenner GJ, Omura T, Samad OA, Costigan M, Inquimbert P, Niederkofler V, Salie R, Sun CC, Lin HY, Arber S, Coppola G, Woolf CJ, Samad TA: The BMP coreceptor RGMb promotes while the endogenous BMP antagonist noggin reduces neurite outgrowth and peripheral nerve regeneration by modulating BMP signaling. J Neurosci 2011 31:18391-18400.

36. Jordan J, Bottner M, Schluesener HJ, Unsicker K, Krieglstein K: Bone morphogenetic proteins: neurotrophic roles for midbrain dopaminergic neurons and implications of astroglial cells. Eur J Neurosci 1997, 9:1699-1709.

37. Reiriz J, Espejo M, Ventura F, Ambrosio S, Alberch J: Bone morphogenetic protein-2 promotes dissociated effects on the number and differentiation of cultured ventral mesencephalic dopaminergic neurons. J Neurobiol 1999, 38:161-170

38. Stull ND, Jung JW, lacovitti L: Induction of a dopaminergic phenotype in cultured striatal neurons by bone morphogenetic proteins. Brain Res Dev Brain Res 2001, 130:91-98.

39. Anitha M, Shahnavaz N, Qayed E, Joseph I, Gossrau G, Mwangi S, Sitaraman SV, Greene JG, Srinivasan S: BMP2 promotes differentiation of nitrergic and catecholaminergic enteric neurons through a Smad1-dependent pathway. Am J Physiol Gastrointest Liver Physiol 2010, 298:G375-G383.

40. Ikeda N, Nonoguchi N, Zhao MZ, Watanabe T, Kajimoto Y, Furutama D, Kimura F, Dezawa M, Coffin RS, Otsuki Y, Kuroiwa T, Miyatake S: Bone marrow stromal cells that enhanced fibroblast growth factor-2 secretion by herpes simplex virus vector improve neurological outcome after transient focal cerebral ischemia in rats. Stroke 2005, 36:2725-2730.

41. Viemann D, Goebeler M, Schmid S, Klimmek K, Sorg C, Ludwig S, Roth J: Transcriptional profiling of IKK2/NF-kappa B- and p38 MAP kinasedependent gene expression in TNF-alpha-stimulated primary human endothelial cells. Blood 2004, 103:3365-3373.

42. Hotokezaka H, Sakai E, Kanaoka K, Saito K, Matsuo K, Kitaura H, Yoshida N, Nakayama K: U0126 and PD98059, specific inhibitors of MEK, accelerate differentiation of RAW264.7 cells into osteoclast-like cells. J Biol Chem 2002, 277:47366-47372.

43. Shimo T, Matsumura S, Ibaragi S, Isowa S, Kishimoto K, Mese H, Nishiyama A, Sasaki A: Specific inhibitor of MEK-mediated cross-talk between ERK and p38 MAPK during differentiation of human osteosarcoma cells. J Cell Commun Signal 2007, 1:103-111.

44. Al-Shanti N, Stewart CE: PD98059 enhances C2 myoblast differentiation through p38 MAPK activation: a novel role for PD98059. J Endocrinol 2008, 198:243-252

45. Okura H, Saga A, Fumimoto $Y$, Soeda M, Moriyama M, Moriyama H, Nagai $K$, Lee CM, Yamashita S, Ichinose A, Hayakawa T, Matsuyama A: Transplantation of human adipose tissue-derived multilineage progenitor cells reduces serum cholesterol in hyperlipidemic Watanabe rabbits. Tissue Eng Part C Methods 2011, 17:145-154.

46. Saga A, Okura H, Soeda M, Tani J, Fumimoto Y, Komoda H, Moriyama M, Moriyama H, Yamashita S, Ichinose A, Daimon T, Hayakawa T, Matsuyama A: HMG-CoA reductase inhibitor augments the serum total cholesterollowering effect of human adipose tissue-derived multilineage progenitor cells in hyperlipidemic homozygous Watanabe rabbits. Biochem Biophys Res Commun 2011, 412:50-54.

doi:10.1186/1471-2121-13-21

Cite this article as: Moriyama et al: Human adipose tissue-derived multilineage progenitor cells exposed to oxidative stress induce neurite outgrowth in PC12 cells through p38 MAPK signaling. BMC Cell Biology 2012 13:21. 